\title{
What's attitude should we hold?: The balance between CAM (complementary and alternative medicine) and mainstream medicine.
}

\author{
Tsai-Ju Chien*, MD.MA. MS. Ph.D \\ Division of Hema-Oncology, Department of Internal Medicine, Branch of Zhong-Zhou, \\ Taipei City Hospital, Taipei, Taiwan. \\ Institute of Traditional Medicine, National Yang-Ming University, Taipei, Taiwan \\ No.145, Zhengzhou Rd., Datong Dist., Taipei City 10341, Taiwan (R.O.C.)
}

\begin{abstract}
Asian Journal of Complementary and Alternative Medicine. Volume 09 Issue 1
\end{abstract}
Published on: $17 / 02 / 2021$

*Author for Correspondence: Tsai-Ju Chien*, MD.MA. MS. Ph.D, Division of Hema-Oncology, Department of Internal Medicine, Branch of Zhong-Zhou, Taipei City Hospital, Taipei, Taiwan; E-mail: chientsaiju@gmail.com; Tel: +886979305521

Cite this article as: Chien TJ. What's attitude should we hold?: The balance between CAM (complementary and alternative medicine) and mainstream medicine. Asian Journal of Complementary and Alternative Medicine, Vol 9(1), 20-23:2021.

\section{INTRODUCTION}

In recent decades, more and more people diagnosed with a chronic disease or even cancer as the advance of modern medical technics and detective equipment. Modern people are more awareness of health issues as information exploration and readily available through the internet, media or magazines. In past time, people have not much more choice but obey the physicians' order or prescription. However, modern people are more consciousness and involved in the decision making when facing disease. As has been observed, some patients hesitate about mainstream therapy will seek for complementary and alternative medicine for help. This phenomenon is popular in cancer population or patients who suffered from chronic disease. From the data of National Center for Complementary and integrative medicine, more than 30 percent of adults and about 12 percent of children-use CAM aside from conventional or mainstream medicine, as a result, the controversy between mainstream medicine and CAM has sparked heated debate. Some physicians against their patients are adopting CAM while others not. No matter what position physicians hold; patients always want to know more methods to promote their health well-being; therefore, patients sometimes deny or refuse to tell their doctors that they use CAM beyond mainstream medicine. As serviced as an oncologist, medical physician for more than a decade, many patients I cared in the clinic also seek for CAMs with or without consulting me in advance. No matter the purpose is to against or get a better understanding of CAMs and provide patients with more treatment choices; ultimately, physicians should find a balance between CAM and so-called mainstream medicine so that we can benefit patients more.

The most widely applied CAM includes natural products(Herbs, Traditional Chinese Medicine), Acupuncture, Yoga, Meditation,
Tai-Chi, Qi-Gong, Massage, Homeopathy, special diet. Patients sometimes adopt more than one kind CAM to promote their health.

Patients have mostly consulted about the use of CAM by family medicine doctors and oncologists. Patients ask FM for CAM most out of a mindset with preventive intention, belonging to a healthier population while others consult oncologist might be cancer survivor seeking for further therapeutic agents to combat disease or boost their immunity, preventing disease recurrence.

\section{CONTROVERSIES BETWEEN CAM AND MAINSTREAM MEDICINE}

From decades clinical experience and research in cancer and integrative medicine fields; I found the main controversies lies in several aspects.

1) The lack of research or knowledge to address the possible interactions between some herbs; nutritional supplies and conventional medicine

2) Without sufficient scientific evidence based proof, physicians hesitate to provide or integrate CAM into mainstream medicine.

3) The lack of education in CAM to conventional medicine physicians leads them to hold a skeptical opinion or even unwilling to open to now more about CAM, regardless of conducting relevant studies or discussing with patients more about how to integrate CAM in their mainstream medicine.

4) Patients have no idea whether they should tell their doctors that they have taken CAMs for not all physicians maintain an open mind to something they are uncertain about their efficacy or not belonging to their professionals. 


\section{KNOWLEDGE BARRIER}

The further issues are subject to why physicians reluctant to integrate CAMs in their daily practice as so many people adapt them no matter through professional consultation or not. What's more, how could we address this controversial issue through more clear, open discussions? That is just the point--how to get the balance between CAM and mainstream medicine. In fact, CAMs have certain values and roles in our life, as we consciously or unconsciously applying them in health promotion, such as practicing yoga, taking some herbs or enjoying aromatherapy et al.

When we are not in physicians'role, we do not feel anything wrong by practicing them; we feel challenged when patients ask something beyond our prescriptions, conventional medicine, and the uncertainty contribute to our reluctant. In fact, many types of CAMs origin from ethnic medicine and have existed for thousands of years, such as TCM, acupuncture. Instead of against or put them apart from conventional therapy, it is better to get more knowledge about CAMs or even extract some essence from them, integrating them in conventional medicine through more scientific methods. In light of a more open attitude, I would like to address this issue regarding different kinds CAMs.

\section{HERBAL MEDICINE}

Natural Products, include herbs, Traditional Chinese Medicine. More and more research now targets this field as developing new drugs or finding potential natural herbs have been an urgent issue in modern medical or pharmaceutical field. Take colon cancer for instance, as it incidence rate increasing, some studies (TCM-Kuna-Sin-Yin) conducted targeting to colon cancer reveals that some TCM has valuable roles regarding impairing tumor growth independent of its immune effect ${ }^{1}$ or regulating autonomic nerve system ${ }^{2,3}$. Some decoction(DangguiBuxue Tang) noted as having potential activity as being chemotherapy or radiotherapy sensitizer in colorectal cancer treatment. Regarding climate syndrome, prevailing problems noted in Family Medicine clinic, there are also various studies towards to menopause syndrome, TCM, and natural herbs exert a certain effect on relieving symptom as some patients have a concern about using hormone therapy ${ }^{4,5}$. In an updated review article, several TCM compounds have noted with effect on ameliorating the symptom of Alzheimer's Disease ${ }^{6}$, such as Ginsenoside Rg1, extracted from Radix Ginseng 7 ; Icariin, decreasing levels and the hyper-phosphorylation of tau protein also benefits people suffered from osteoporosis ${ }^{8}$; Tanshinone IIA, extracted from Radix Salviae miltiorrhizae ${ }^{9}$; Huperzine A, extracted from Huperzia Serrata ${ }^{10}$. All of these exert a different effect through various molecular pathways. As has been listed above, I just want to raise the attention and awareness from mainstream physicians that it is a trend to absorb more knowledge from CAMs for their potential in both clinical and laboratory findings. As clinical physicians, we can directly observe the patient's response; thus, if clinical physicians could get involved more in conducting trials towards these novel agents; I am convinced more novel, effective drugs could be developed. Undoubtedly, it takes time, effort and cooperation between industries; nevertheless, it is a trend we can not avoid facing it, so why not involve more and hold an open yet objective attitude?

\section{ACUPUNCTURE}

As for acupuncture, an old art of Traditional Chinese Medicine, has been recognized as World Intangible Cultural Heritage of Humanity by United Nations Educational, Scientific, and Cultural Organization (UNESCO) since 2003. Acupuncture most applied in chronic pain ${ }^{11}$, poor quality of sleep ${ }^{12,13}$, psychologic problems ${ }^{14}$, hot flush ${ }^{15,16}$ for its safety and non-invasive characters. Compared with herbs and TCM, acupuncture has been much more accepted and adapted in western countries for its mechanism of electrophysiology has been earlier defined ${ }^{17,18}$. The base of meridian theory somehow compatible with electrophysiology response noted in current studies ${ }^{3,19}$. Physicians mostly have not as many criticisms on acupuncture as for herbs, for acupuncture does not belong to medication, won't have a drug's interactions with conventional medicine, yet suggestion holds towards to patients with inflammation or open wounds.

\section{YOGA, TA-CHI, MEDITATION}

Regarding Yoga, Ta-Chi, meditation, massage, more physicians are willing to encourage patients to participate in these activities though they are not sure the efficacy on the diseases. Research indicate that yoga; Ta-Chi could improve patients' life quality and sleep ${ }^{20}$; meditation may exert a positive effect on not only psychologic but also physiologic problems $\mathrm{s}^{21,22}$. These oriental theories based psychiatric- medicine intrigued some scientists and mainstream physician for meditation stimulating alpha waves in EEG measurements ${ }^{23}$. Some western experts recently indicated that contrary to the belief that Mindfulness only origins from Buddhist and Hindu; it is also rooted in Jewish, Islamic and Christian religions; mindfulness can be viewed as a universal human ability embodied to foster clear thinking and open-heartedness and in turn, promote physiological and physiological health ${ }^{24}$. The puzzles may not be answered immediately, and some issues lie not only in scientific fields. Therefore, physicians better hold an opening, observative, and more objective attitude when patients ask opinions for taking part in these activities. 


\section{HOMEOPATHY}

Homeopathy, while surrounded by debates of ethnic issues, has been questioned its safety and efficacy. As some research reveal that it may have a placebo effect; a recently published paper provides a new viewpoint: instead arguing its feasibility, it is better discussing a framework to establish the extent to which the dominant (medical) culture should tolerate non-dominant health practices such as homeopathy ${ }^{25}$. What physicians are whether patients asked well-trained homeopathic practitioners to carry out such a task. If any positive effects were noted, it hard to define whether the efficacy comes from conventional therapy or synergic effect of concurrent homeopathy. The uncertainty may somehow make dominant physicians feel confused in general medicine department if they were not involved in a trial or well conscious of their patients are undergoing such complementary.

\section{METHODOLOGY BARRIER}

As has been described, they are many problems existing in CAMs; a modern scientific methodology can not totally answer some for they originally belong to different categories or culture, such as TCM, meditation, yoga. As it is not fair to judge an art with scientific viewpoint; it is also hard to prove its efficacy in health self-healing. Before more suitable methodology designed or more evidence-based studies providing physicians a whole picture, I sincerely suggest relevant institutions could emphasize more on education about CAMs towards to both doctors and the public. As a hematooncologist, I could not deny the potential and positive effect I observed in clinic though some phenomena still lack evidencebased proof. I become more humble, facing the greatness of life in front of the boon of creation. It is undoubtedly better holding a conservative and rational as any physician could have about the practice of complementary and alternative medicine, acknowledging we should be more aware of the importance of the mind-body relationship in well -being. What's more, we could also increase CAMs lectures in medical education, so that infilling knowledge in future physicians and encouraging the willingness to entertain nonconventional modalities with informed; so far, NCCIH has been promoting this campaign for decades. Another rationale is if physicians can accept CAMs will always exist as well as patients have right to try integrative medicine; then it is better to encourage CAMs practitioners; to register and conduct well-trained programs; otherwise; who should be responsible for it if CAMs are not ruled by government based health administrations?

\section{CONCLUSION}

To sum up, it is the responsibility of doctors to involve the patient's health, to deliver the health care that draws on the best of the scientific approach to medicine, but not exclude things because we do not understand them. I sincerely hope and raise the general physicians áttention to enable patients to benefit from the full array of modalities which can be shown to improve care'and participate in more trials to get deeper investigations in relevant issues and expect CAMs practice could be more regularized and integrated as well.

\section{REFERENCES}

1. Wilmore JH (1997) Physiology of sports. DL Church.

2. Czuba M, Wilk R, Karpinski J, Chalimoniuk M, Zajac A, et al. (2017) Intermittent hypoxic training improves anaerobic performance in competitive swimmers when implemented into a direct competition mesocycle. Plos One 12: e0180380.

3. Tolstopyatov IA, Redi EV, Ivanitsky VV, Zakharova LV (2018) Analysis of Underwater Swimming World Record Gains Regularities (Finswimming).

4. Kononova EV (2015) Adaptation of children and youth to modern socio-economic conditions on the basis of healthy saving technologies. Abakan 13: 148-150.

5. Folland JP, Williams AG (2010) The adaptations to strength training: Morphological and neurological contributions to increased strength. Sports Med 37: 145-168.

6. Maksimov AL (2012) Features of the functional state in young men from Magadan, depending on the type of constitution 12: 25-30.

7. Martirosov G (1977) Major Contemporary issues in sports. Sports Science and Physical Education 2: 8-29.

8. Martirosov JG (1968) Morphofunctional organization and sports.

9. Moskovchenko ON (2005) The teleological approach to the selection and management of the training of Greco-Roman wrestlers at the stage of advanced specialization: Monograph. Ipc Kgtu 2005: 158.

10. Moskovchenko ON (2014) Underwater sports and diving: Study guide.

11. Moskovchenko ON (2019) World records in finswimming sport: Growth profiling analysis. Theory and Practice of Physical Culture 969: 70-72.

12. Mjasnikova TI (2016) Evaluation of the fitness of qualified swimmers-divers using standard loads of varying intensity. Ural Federal University the first President of Russia V.N. Yeltsin 2016: 267-272.

13. SimakovaAN (2016) Morphological and functional characteristics of gymnasts aged 8-14 years of the city of Krasnoyarsk/Abstract of the thesis of Candidate of Medical Sciences Simakova Lyudmila Nikolaevna. KGMA 2006: 27.

14. Tjo S (1997) Sports morphology of highly qualified weightlifters // Material konferencii 9-12 ijunja 1997g. Krasnoyarsk//Aktual'nye voprosy biomedic.

15. Shumakov AV (1997) Control of the training process of wrestlers according to model characteristics. Physical culture education in Siberia 1: 82 . 
16. Bendikova E (2014) Lifestyle, Physical and Sports Education and Heaith Benefits of Physical Activity. European Researcher 69: 343-348.

17. Cekovik N, Dordevic M, Stefanovic N (2013) Somatotype analysis of the first-year students of the faculty of sport and physical education in Nis. XVI Scientific Conference "FIC COMMUNICATION 2013" in physical education, sport and recreation and I International Scientific Conference 23: 369-376.

18. Gaurav V, Singh A (2014) Anthropometric characteristics of Indian volleyball players in relation to their performance level. Turkish Journal of Sport and Exercise 16: 87-89.
19. Moskovchenko O Ivanitsky V, Zakharova L, Tolstopyatov I, Tatiana K, et al. (2018) Morphofunctional markers of kinetic aptitude in a sport selection system. Journal of Physical Education and Sports 18: 670-676.

20. Moskovchenko O (2011) Psycho-Physiological Types of Adaptation in the Assessment of Professional and Sport Selection. Journal of Siberian Federal University. Humanities \& Social Sciences 9: 1288-1300.

21. Pordigalo L, Iermakov S, Potop V, Romanenko V, Boychenko N, et al. (2017) Special Aspects of Psychophysiological Reactions of Different Skillfulness Athletes, Practicing Martial Arts. Journal of Physical Education and Sport 17: 519-526. 